\title{
A new surgical approach for punctal occlusion using fibrous tissue from under the lacrimal caruncle
}

This article was published in the following Dove Press journal:

Clinical Ophthalmology

\author{
Norihiko Yokoi' \\ Aoi Komuro' \\ Chie Sotozono' \\ Shigeru Kinoshita ${ }^{2}$ \\ 'Department of Ophthalmology, \\ Kyoto Prefectural University of \\ Medicine, Kyoto, Japan; '2Department \\ of Frontier Medical Science and \\ Technology for Ophthalmology, \\ Kyoto Prefectural University of \\ Medicine, Kyoto, Japan
}

Purpose: Surgical punctal occlusion is indispensable for the treatment of severe dry eye in cases where punctal-plug insertion is not applicable due to an enlarged or deformed punctum. However, permanent punctal occlusion is difficult in some cases. In our aim to establish a more reliable punctal occlusion, we have devised a new surgical approach for punctal occlusion.

Patients and methods: This study involved 20 puncta of 12 eyes of 12 patients ( 1 male and 11 females; mean age: 65.2 years) with severe aqueous-tear-deficient dry eye. A new surgical procedure for punctal occlusion using fibrous tissue from under the lacrimal caruncle into the diathermy-induced deepithelialized canaliculus as supporting tissue for punctal closure was performed. In all patients, the assessment of eye symptoms, as well as the condition of punctal occlusion by slit-lamp biomicroscopy, tear volume (tear-meniscus radius [TMR] measurement by meniscometry), the condition of precorneal tear film (graded by interferometry [IG]), measurement of fluorescein breakup time (FBUT), and scoring of ocular surface staining (fluorescein score of area [FSA] and density [FSD], and lissamine green score [LGS]) were performed, and the preoperative and 6-month-postoperative values were compared.

Results: In regard to the postoperative improvement of symptoms, 11 patients showed remarkable improvement, 1 patient showed improvement, and no reopening of the closed punctum was found in any patient. Test values were all significantly improved post surgery (all: $P<0.05$ ) as compared to those prior to surgery (respective values [mean $\pm \mathrm{SD}$ ], and the pre- and postoperative $P$-values were: TMR (mm) [0.18 $\pm 0.08 ; 0.56 \pm 0.28, P=0.002]$, IG [4.3 $\pm 0.9 ; 2.7 \pm 0.8, P=0.009]$, FBUT [0.4 $\pm 0.6 ; 4.1 \pm 2.9, P=0.004]$, FSA [1.6 $\pm 0.7 ; 0.7 \pm 0.9, P=0.03]$, FSD [2.7 $\pm 0.7 ; 0.6 \pm 0.7$, $P=0.003]$, and LGS $[5.1 \pm 2.7 ; 1.1 \pm 2.1, P=0.005])$. Moreover, no postoperative complications were observed.

Conclusion: The findings of this study showed that our novel surgical procedure for punctal occlusion is highly successful and that it results in improved and more complete punctal occlusion.

Keywords: surgical punctal occlusion, aqueous-tear-deficient dry eye, lacrimal caruncle

\section{Introduction}

Sjögren's syndrome (SS)-related aqueous-tear-deficient dry eye (ATDDE) ${ }^{1}$ sometimes results in severe superficial punctate keratopathy and/or persistent corneal filaments that can lead to severe ocular discomfort and/or pain. In such severe cases, since medical treatment involving the topical instillation of artificial tears or hyaluronic acid eye drops has some limitations, blocking of the lacrimal canaliculi and/or puncta is often chosen as the appropriate alternative treatment. ${ }^{2,3}$ For blocking of the lacrimal canaliculi and/or puncta, punctal plugs are generally used as the initial treatment, ${ }^{3}$ since in most cases that procedure can be reversed. However, in cases where the use of punctal plugs is not applicable, eg, when repeated plug extrusion results in the punctum becoming
Correspondence: Norihiko Yokoi Department of Ophthalmology, Kyoto Prefectural University of Medicine, 465 Kajii-cho, Hirokojiagaru, Kawaramachi-dori, Kamigyo-ku, Kyoto 602084I, Japan

Tel $+817525 I 5578$

Fax $+8 \mid 7525$ I 5663

Email nyokoi@koto.kpu-m.ac.jp
Clinical Ophthalmology 2018:12 463-472 (c) (1) (\$) 2018 Yokoi et al. This work is pulished and licensed by Dove Medical Press Limited. The full terms of this license are available at https://www.dovepress.com/terms.php

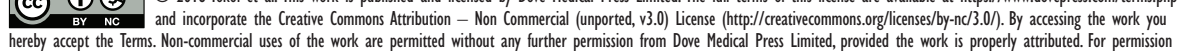
for commercial use of this work, please see paragraphs 4.2 and 5 of our Terms (hitps://www.doverpess.comm/terms.php).
Dovepress

http://dx.doi.org/10.2147/OPTH.S155209 
so enlarged that no appropriate plug size is available ${ }^{4}$ or when the reopened canaliculus and/or punctum is deformed due to the failure of earlier blocking methods, ${ }^{2}$ a more reliable blocking method is necessary to improve the patient's symptoms. Various methods of punctal occlusion have previously been reported, ${ }^{2}$ however, some surgical methods, such as dacryocystectomy, nasolacrimal duct occlusion, or canalicular excision, ${ }^{5-7}$ are relatively invasive and sometimes difficult to reverse. Cauterization methods such as thermal cautery, ${ }^{8-10}$ diathermy, ${ }^{11}$ laser surgery, ${ }^{12}$ canalicular ligation, ${ }^{13}$ removal of punctal and canalicular epithelium followed by sutures, ${ }^{14}$ or a punctal patch ${ }^{10}$ are reportedly simple and less invasive, yet those methods alone may produce varied and unpredictable results, often leading to a reopening of the once-closed punctum and/or canaliculus. ${ }^{8-10,12,14}$

The purpose of this present study was to investigate the efficacy of our newly developed surgical procedure to overcome the recanalization of once-surgically occluded punctum and/or canaliculus, and to establish a standardized reliable method for punctal occlusion.

\section{Patients and methods}

\section{Subjects}

This study was approved by the Institutional Review Board of Kyoto Prefectural University of Medicine, Kyoto, Japan and was conducted in accordance with the tenets set forth in the Declaration of Helsinki. Prior written informed consent was obtained from all subjects after they received a detailed explanation of the nature of the study and possible consequences associated with their participation.

This study involved 20 puncta of 12 eyes of 12 patients (ie, 2 puncta of 1 eye of 1 male patient and 18 puncta of 11 eyes of 11 female patients). The mean patient age was $65.2 \pm 14.3$ years (mean \pm SD) (29-82 years). According to the diagnostic criteria described below, all 12 patients had ATDDE. Of those 12 patients, 7 patients had SS dry eye (ie, 3 cases of primary SS and 4 cases of secondary $\mathrm{SS}$, with the diagnosis of SS based on the criteria of Fox and associates), ${ }^{15} 3$ patients had non-SS dry eye, 1 patient had ocular cicatricial pemphigoid, and 1 patient had graftversus-host disease. As shown in Table 1, of the 18 puncta of the 12 eyes enrolled, 7 puncta of 4 eyes had undergone frequent punctal-plug extrusion only (Cases 8, and 10-12), and 13 puncta of 8 eyes had undergone repeated punctal-plug extrusion and repeated failure of conventional electrodiathermy ${ }^{2}$ and sutures (Cases 1-7, and 9). Five puncta were left untreated since they were stably closed due to punctal-pluginduced granulation (Case 8) or due to the fact that the punctal plug was properly fitted (Cases 1-3, and 12) (Table 1).

Prior to enrollment, each subject underwent tear tests including the Schirmer's 1 test $(\mathrm{ST} 1)^{16}$ (abnormal value: $\leq 5 \mathrm{~mm} /$ minute), measurement of fluorescein breakup time $\left(\right.$ FBUT) ${ }^{17}$ (abnormal value: $\leq 5$ seconds), scoring of corneal fluorescein staining (FS) ${ }^{18}$ (abnormal FS scores: $\geq A 1 D 1$ [A and D representing area and density, respectively], respectfully graded from 0 [none] to 1 [mild],

Table I Data summary of the 12 patients who underwent our newly developed surgical method for punctal occlusion

\begin{tabular}{|c|c|c|c|c|c|c|c|c|c|c|c|c|c|c|c|c|c|c|}
\hline \multirow{3}{*}{$\begin{array}{l}\text { Case } \\
\text { no. }\end{array}$} & \multirow{3}{*}{$\begin{array}{l}\text { Age } \\
\text { (years) }\end{array}$} & \multirow[t]{3}{*}{ M/F } & \multirow[t]{3}{*}{ BD } & \multirow{3}{*}{$\begin{array}{l}\text { Pre- } \\
\text { STI }\end{array}$} & \multirow[t]{3}{*}{ OpP } & \multicolumn{2}{|c|}{ TMR (mm) } & \multicolumn{2}{|l|}{ IG } & \multicolumn{2}{|c|}{ FBUT } & \multicolumn{4}{|l|}{ FS } & \multicolumn{2}{|l|}{ LGS } & \multirow{3}{*}{$\begin{array}{l}\text { OP } \\
\text { (months) }\end{array}$} \\
\hline & & & & & & \multirow[t]{2}{*}{ Pre } & \multirow[t]{2}{*}{ Post } & \multirow[t]{2}{*}{ Pre } & \multirow[t]{2}{*}{ Post } & \multirow[t]{2}{*}{ Pre } & \multirow[t]{2}{*}{ Post } & \multicolumn{2}{|c|}{ Pre } & \multicolumn{2}{|c|}{ Post } & \multirow[t]{2}{*}{ Pre } & \multirow[t]{2}{*}{ Post } & \\
\hline & & & & & & & & & & & & $A$ & D & A & D & & & \\
\hline I & 78 & $\mathrm{~F}$ & S-SS & 3 & RE L & 0.12 & 1.23 & 5 & 2 & 0.7 & 4 & 2 & 3 & 0 & 0 & 5 & 0 & 12.3 \\
\hline 2 & 82 & $\mathrm{~F}$ & OCP & 0 & RE U & 0.32 & 0.39 & 5 & 2 & 0 & 2.7 & 2 & 3 & I & 2 & 9 & 2 & 10.3 \\
\hline 3 & 55 & $\mathrm{~F}$ & Non-SS & 5 & RE U & 0.19 & 0.52 & 4 & 2 & 2 & 5.3 & I & 3 & 0 & 0 & I & 0 & 9.25 \\
\hline 4 & 61 & $\mathrm{~F}$ & Non-SS & I & LE U\&L & 0.24 & 0.47 & 4 & 2 & 0 & 1 & 3 & 2 & 3 & I & 7 & 7 & 9.25 \\
\hline 5 & 29 & $M$ & GVHD & 4 & LE U\&L & 0.17 & 0.36 & 3 & 3 & 0 & 1.3 & 2 & 3 & I & I & 5 & 0 & 9 \\
\hline 6 & 59 & $\mathrm{~F}$ & Non-SS & 0 & RE U\&L & 0.09 & 0.34 & 5 & 2 & 0 & 3.3 & 2 & 3 & 0 & 0 & 4 & 0 & 8 \\
\hline 7 & 63 & $\mathrm{~F}$ & S-SS & 2 & LE U\&L & 0.33 & 0.88 & 5 & 3 & 0 & 9.3 & 2 & 3 & 0 & 0 & 8 & 0 & 7.3 \\
\hline 8 & 80 & $\mathrm{~F}$ & P-SS & 1 & LE L & 0.13 & 0.51 & 5 & 4 & 0 & 7.7 & I & 2 & 1 & I & 8 & 0 & 6 \\
\hline 9 & 67 & $\mathrm{~F}$ & P-SS & 4 & RE U\&L & 0.12 & 0.68 & 3 & 3 & 0 & 1 & I & 3 & I & I & 6 & 3 & 13 \\
\hline 10 & 64 & $\mathrm{~F}$ & P-SS & 2 & LE U\&L & 0.17 & 0.72 & 4 & 3 & 1 & 6 & I & 3 & 0 & 0 & 5 & 0 & 6.7 \\
\hline 11 & 74 & $\mathrm{~F}$ & S-SS & 4 & LE U\&L & 0.15 & 0.29 & 5 & 4 & 0.3 & 6 & I & I & 0 & 0 & I & 0 & 6 \\
\hline 12 & 70 & $\mathrm{~F}$ & S-SS & 2 & LE U\&L & 0.12 & 0.34 & 3 & 2 & 1 & 1 & $\mathrm{I}$ & 3 & 1 & $\mathrm{I}$ & 2 & 1 & 6 \\
\hline Mean & 65.2 & & & 2.3 & & 0.18 & 0.56 & 4.3 & 2.7 & 0.4 & 4.1 & 1.6 & 2.7 & 0.7 & 0.6 & 5.1 & I.I & 8.6 \\
\hline SD & 14.3 & & & 1.7 & & 0.08 & 0.28 & 0.9 & 0.8 & 0.6 & 2.9 & 0.7 & 0.7 & 0.9 & 0.7 & 2.7 & 2.1 & \\
\hline \multicolumn{6}{|l|}{$P$-value } & \multicolumn{2}{|c|}{0.002} & \multicolumn{2}{|c|}{0.009} & \multicolumn{2}{|c|}{0.004} & \multicolumn{2}{|c|}{0.030} & \multicolumn{2}{|c|}{0.003} & \multicolumn{2}{|c|}{0.005} & \\
\hline
\end{tabular}

Abbreviations: M/F, male/female; BD, background disease; S-, secondary; P-, primary; SS, Sjögren's syndrome; OCP, ocular cicatricial pemphigoid; GVHD, graft-versus-host disease; STI, Schirmer I test; OpP, operated punctum; RE, right eye; LE, left eye; U, upper; L, lower; TMR, tear meniscus radius; IG, interference grade; FBUT, fluorescein breakup time; FS, fluorescein score; A, area; D, density; LGS, lissamine green score; OP, observation period. 
2 [moderate], and 3 [severe]), and ocular-surface staining by lissamine green ${ }^{19}$ (LGS: an abnormal score for lissamine green being $\geq 3$, based on the van Bijsterveld criteria for rose bengal staining). ${ }^{20}$

The ATDDE subjects enrolled in this study met the following inclusion criteria: an abnormal ST1 value and abnormal FS or LGS; all eyes also showing abnormal FBUT values (summarized in Table 1). None of the eyes of the enrolled patients could be treated through the use of eye drops (ie, including preservative-free artificial tears, steroids, hyaluronic acid, or combinations of these), and had therefore received treatment for punctal occlusion. On the pre- and postoperative examination days, all subjects were requested to not use their usual eye drops within 1-hour prior to the examination.

\section{Evaluation of tear volume}

In this study, to evaluate the effect of our newly developed surgical punctal occlusion on the retention of tears and/or artificial tears over the ocular surface, the pre- and postoperative radii of the central portion of the lower tear meniscus radius (TMR, mm) were compared. The tear meniscus represents $75 \%-90 \%$ of the total tear volume ${ }^{21}$ and the TMR value reflects the total tear volume over the ocular surface, ${ }^{22}$ so video-meniscometry ${ }^{22-25}$ was used to measure that value. Video-meniscometry involves the projection of an illuminated target consisting of a pair of black and white stripes onto the meniscus. A reflected image of that target is then digitally captured in real time and the TMR value is subsequently calculated via the "concavemirror" formula.

\section{Evaluation of the precorneal tear film (TF)}

In this study, we have also investigated the condition of the precorneal TF before and after surgery via the use of a video-interferometer (DR-1 ${ }^{\mathrm{TM}}$; Kowa Co. Ltd., Tokyo, Japan). ${ }^{26}$ This instrument is equipped with both low- and high-magnification viewing modes which allow observation of a $6.8 \mathrm{~mm}$ (vertical) $\times 8.8 \mathrm{~mm}$ (horizontal) or $2.3 \mathrm{~mm}$ (vertical) $\times 3.2 \mathrm{~mm}$ (horizontal), respectively, rectangular area of the center of the cornea. In this study, the high magnification mode was chosen to obtain information about the TF lipid layer interference, as interferometers provide quantitative information on the thickness of the lipid layer ${ }^{27}$ and postblink lipid layer spread..$^{28}$ However, in this study, both pre- and postoperative interference patterns were evaluated qualitatively using the high magnification mode according to our interferometry grading (IG) system. ${ }^{26}$

\section{Surgical steps of the newly developed punctal occlusion and postoperative care}

Our newly developed surgical technique was aimed at producing a complete and permanent punctal occlusion, with no reopening of the puncta post surgery. To achieve that goal, a small amount of solid fibrous tissue was obtained from under the lacrimal caruncle and was inserted into the deepithelialized canaliculus to keep the closed punctum permanently fixed. However, with that aspect aside, the steps of our surgery follow those that have been previously established. ${ }^{11}$ The anesthesia steps of our new surgical procedure were as follows. First, topical anesthetic eye drops using $0.4 \%$ oxybuprocaine (Benoxinate Hydrochloride 0.4\%; Santen Pharmaceutical Co. Ltd., Osaka, Japan) were instilled. Next, pressure anesthesia ${ }^{29}$ was applied for 10 minutes to relieve pain at the time of injecting the local anesthesia (in accordance with the reported technique involving the use of a cotton swab soaked with 4\% lidocaine [Xylocaine ophthalmic solution 4\%; AstraZeneca K.K., Osaka, Japan], with repeated instillation to keep the swab wet). Finally, additional local anesthesia was applied around the punctum using $2 \%$ lidocaine (Xylocaine injection 2\%; AstraZeneca).

The remaining surgical steps were as follows and are shown in Figure 1A and B. First, diathermy was gently applied to approximately the first $2 \mathrm{~mm}$ of the canalicular epithelium below the punctum (ie, the vertical portion of the canaliculus) using a diathermy unit (OM-401S; Tagawa Electric Research Institute Ltd., Chiba, Japan) equipped with a newly designed monopolar electric needle. ${ }^{11}$ Next, the diathermy-induced heat-injured epithelium was removed from the canaliculus using a hand-held motor burr grinder for the corneal rust ring ${ }^{11}$ (S-226 Handy Micromotor; Inami Co. Ltd., Tokyo, Japan). A 2-mm incision was then made along the temporal edge of the lacrimal caruncle, with fibrous tissue being drawn from under the caruncle, excised, and cut in half in cases where it was necessary to occlude both the upper and lower puncta. A small excised portion of the medial rectus capsulopalpebral fascia $(\mathrm{CPF})$ was then inserted into the deepithelialized canaliculus and fixed with one stitch of 10-0 nylon placed through the excised fibrous tissue between each contralateral edge of the punctum, with the size of the tissue for excision being determined in accordance with the beforehand-measured size of the punctum. Finally, additional 10-0 nylon stitches were placed in a cross pattern to fix the fibrous tissue tightly against the deepithelialized canalicular wall, thus completely closing the punctum; the number of sutures (which ranged from 2 to 5 ) was determined according to the size of punctum. 
A
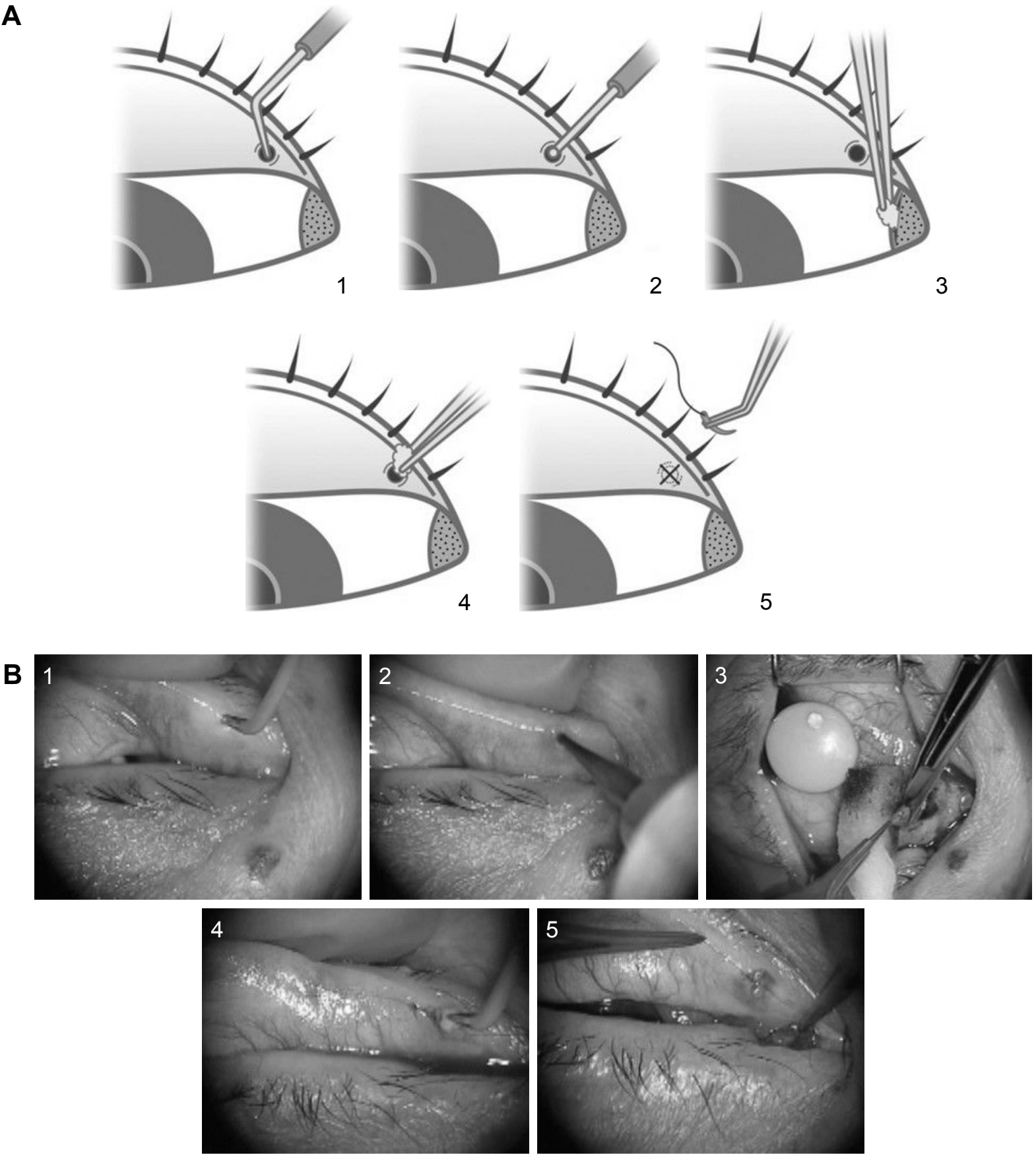

Figure I Surgical steps of our newly developed punctal occlusion method ([A]. schematic diagram, [B]. surgical photo).

Notes: (I) diathermy, (2) deepithelialization with a hand-held motor burr grinder, (3) removal of a section of the medial rectus capsulopalpebral fascia (CPF) from under the lacrimal caruncle, (4) insertion of the CPF tissue into the deepithelialized canaliculus, (5) anchoring and additional sutures placed to fix the CPF tissue against the deepithelialized canalicular wall to close the punctum.

Following surgery, the patients were instructed to wear an eye patch during sleeping hours for 1 week to prevent any accidental breakage of sutures. The sutures were removed when they became loose, yet those covered by conjunctiva were left in place. Until 4-weeks postoperative, $0.1 \%$ fluorometholone (Fluorometholone 0.1\%; Santen Pharmaceutical) and $0.5 \%$ levofloxacin (Cravit ${ }^{\circledR}$ Ophthalmic Solution; Santen Pharmaceutical) were instilled twice daily.
In addition, preservative-free artificial tears containing $0.1 \%$ $\mathrm{KCl}$ and $0.4 \% \mathrm{NaCl}$ (Soft Santear ${ }^{\circledR}$; Santen Pharmaceutical) were instilled six-times daily to wash out the mucus component of the tears.

\section{Postoperative follow-up}

To evaluate the effectiveness of our novel surgery, all patients underwent follow-up examinations at 2-days, 2-weeks, and 
1-, 3-, and 6-months postoperative. At each follow-up examination, TF examination by meniscometry, interferometry, and the measurement of TF breakup time, as well as the fluorescein and lissamine green staining scores (focused on ocular surface epithelium) were examined and compared with those obtained prior to surgery. In addition, each follow-up examination included examination of the operated punctum via slit-lamp microscopy. Slit-lamp examinations of the cornea using fluorescein were performed up to 13-months postoperative, and were continued from that point forward in some cases to confirm good closure of the punctum. Moreover, the improvements of subjective symptoms (dry-eye sensation and foreign body sensation) were self-graded by the patients into one of the following: remarkably improved, improved, unchanged, and deteriorated.

\section{Statistical analysis}

For statistical analysis, comparison between the results of the examinations performed at pre- and 6-months postoperative were made. FBUT, FS, LGS, and IG were compared using the Wilcoxon test. For comparison of the TMR, a paired $t$-test was used. A $P$-value of $\leq 0.05$ was considered statistically significant.
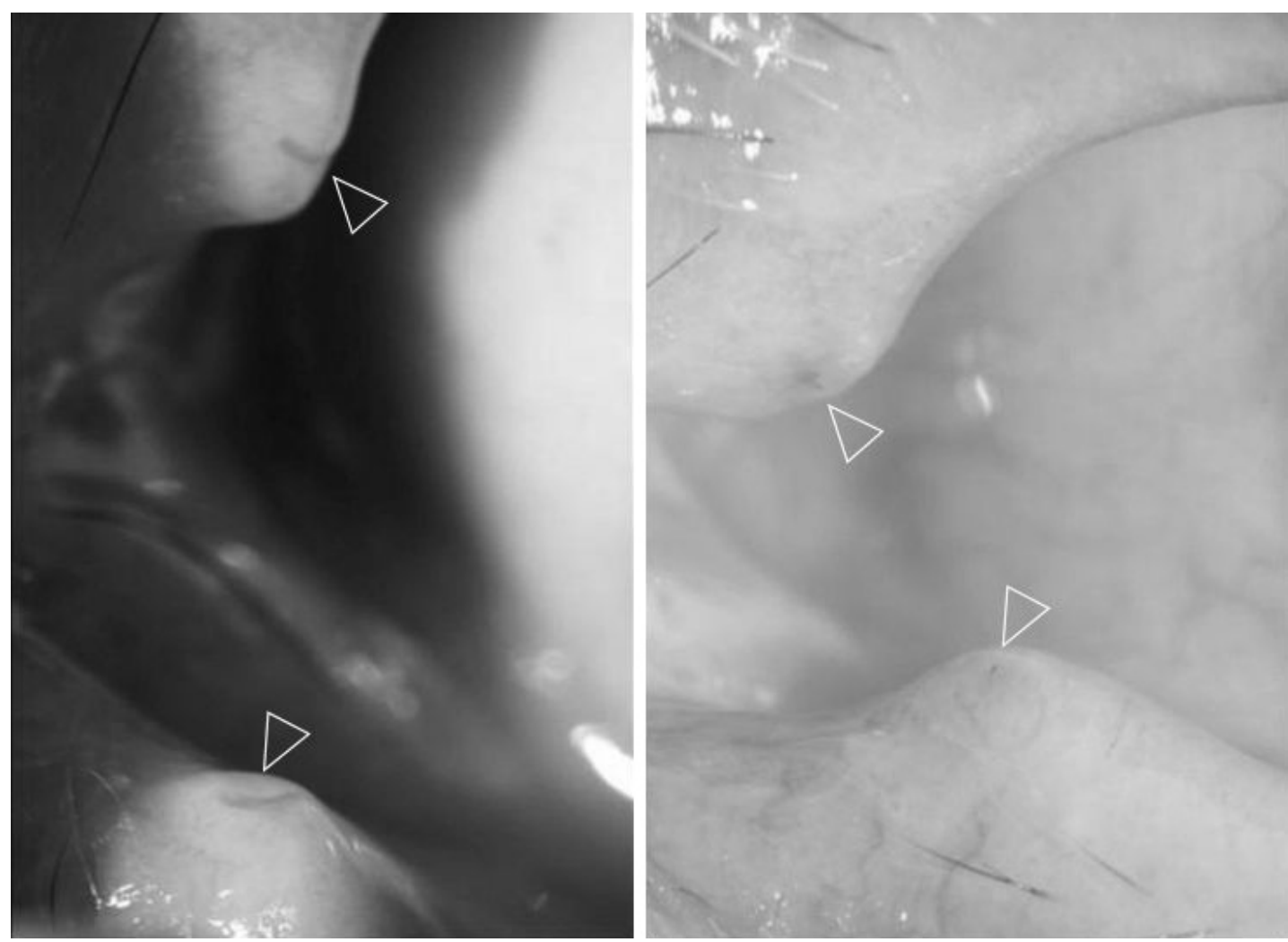

Figure 2 Representative case in which puncta are clearly visible (Case 12 in Table I).

Note: Images showing the enlarged puncta due to frequent punctal-plug extrusion that appeared prior to the patient undergoing our newly developed surgical method (left, arrowheads) and at 6-months postoperative (right, arrowheads).

\section{Results \\ Slit-lamp examination findings}

During the postoperative follow-up period (mean postoperative follow-up period: 8.6 months; range: $6-13$ months), it was found that complete punctal occlusion by the conjunctiva was maintained in all cases. Postoperative inflammation around the punctum was observed, but it disappeared within 1-month postoperative. A representative example of a closed puncta at 6-month postoperative is shown in Figure 2.

Individual results of the examinations, including tear volume and the condition of the precorneal TF, as well as ocular surface staining on the last examination prior to surgery and at 6-months postoperative are summarized in Table 1 and Figure 3 (the statistical analyses in Table 1 and Figure 3 are summarized below). Representative photographs obtained prior to and after surgery (Case 12 in Table 1) are shown in Figure 2, and representative cases (Cases 5 and 7 in Table 1) are shown in Figures 4 and 5, respectively.

\section{Analysis of tear volume and precorneal TF}

The findings of the meniscometry examinations revealed that the mean TMR (mm) at 6-months postoperative was statistically greater than that of prior to surgery $(P=0.002)$; 
A

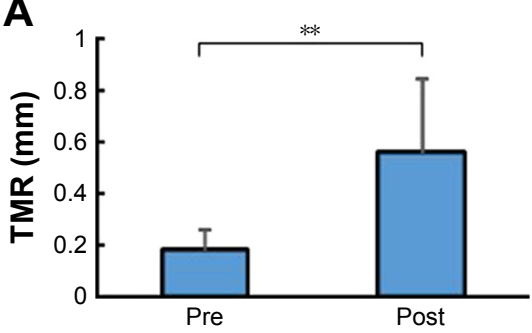

D

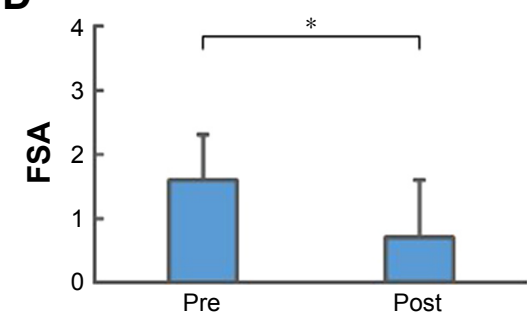

B

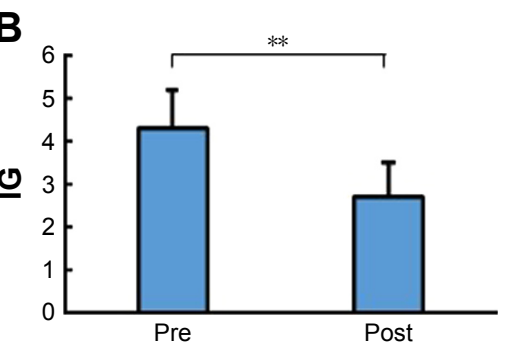

E

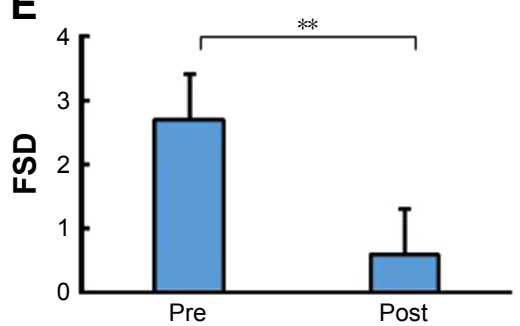

C

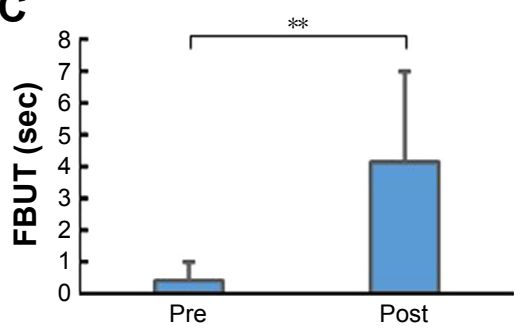

$\mathbf{F}$

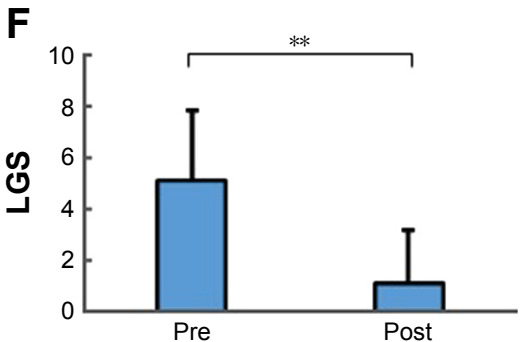

Figure 3 Changes in ocular parameters pre and post surgery.

Notes: (A) Tear meniscus radius (TMR). (B) Interference grade (IG). (C) Fluorescein breakup time (FBUT). (D) Fluorescein scores of area (FSA). (E) Fluorescein scores of density (FSD). (F) Lissamine green scores (LGS). $* P<0.05$, $* * P<0.01$.

$0.18 \pm 0.08$ [mean $\pm \mathrm{SD}$ ] and $0.56 \pm 0.28$, respectively). Moreover, interferometry findings showed that the mean IG at 6-months postoperative was statistically smaller than that at before surgery $(P=0.009) ; 4.3 \pm 0.9[$ mean $\pm \mathrm{SD}]$ and $2.7 \pm 0.8$, respectively). In addition, the FBUTs (in seconds) at 6-months postoperative were statistically longer than those at before surgery $(P=0.004) ; 0.4 \pm 0.6[$ mean $\pm \mathrm{SD}]$ and $4.1 \pm 2.9$, respectively).

\section{Analysis of ocular surface staining}

In regard to the corneal fluorescein staining, a significant improvement in FS was obtained in both A (area) and D (density) in all 12 cases at 6-months postoperative (A: $0.7 \pm 0.9$ [mean $\pm \mathrm{SD}$ ]; D: $0.6 \pm 0.7)$ as compared to that of before surgery (A: $1.6 \pm 0.7 ; \mathrm{D}: 2.7 \pm 0.7)(P=0.030$ and 0.003 , respectively). In addition, in the ocular surface lissamine green staining, a significant improvement in LGS was obtained with reference to the van Bijsterveld classifications (pre surgery: 5.1 \pm 2.7 ; post surgery: $1.1 \pm 2.1, P=0.005) .{ }^{20}$

In all cases, the condition of the eyes were maintained postoperatively using only eye drops, and except for minor linear scarring at the conjunctival incision adjacent to the lacrimal caruncle, no postoperative complications were observed.

\section{Improvement in symptoms}

At 6-months postoperative, the subjective symptoms of the patients were remarkably improved in 11 patients, and also improved in 1 patient whose unilateral punctum was closed incompletely with a punctal plug due to the deformed punctum caused by repeated punctal surgery; none of the patients reported unchanged or deteriorated symptoms, or complained of symptomatic epiphora.

\section{Discussion}

Numerous methods have been developed for permanent punctal occlusion, including thermal cautery, ${ }^{8-10}$ diathermy, ${ }^{11}$ and laser surgery, ${ }^{12}$ as well as other techniques such as canalicular ligation, ${ }^{13}$ removal of punctal and canalicular epithelium followed by sutures, ${ }^{14}$ or the use of a punctal patch. ${ }^{10}$ However, none of these methods have been able to achieve a long-term postoperative $100 \%$ success rate.

In developing our new method of long-term stable punctal occlusion, we tried to focus our attention on the causes of recanalization. In our opinion, recanalization is caused by mechanical friction associated with blinking, the generation of suction in the lacrimal system caused by blinking, and in particular, the tendency of the surgically deformed mucosal ring $^{2}$ to resume its normal shape. Since our method does not rely on the deformation of the mucosal ring, the stress related to a surgically deformed ring is avoided. Furthermore, in our method, we believe the insertion of fibrous tissue from under the caruncle not only allows the mucosal ring to maintain its shape but also helps to support and stabilize the occluded punctum and lessen the chance of recanalization brought on by the mechanical stress of blinking and the generation of suction in the lacrimal system.

The findings in this study demonstrated that our newly developed surgery for punctal occlusion is both safe and 

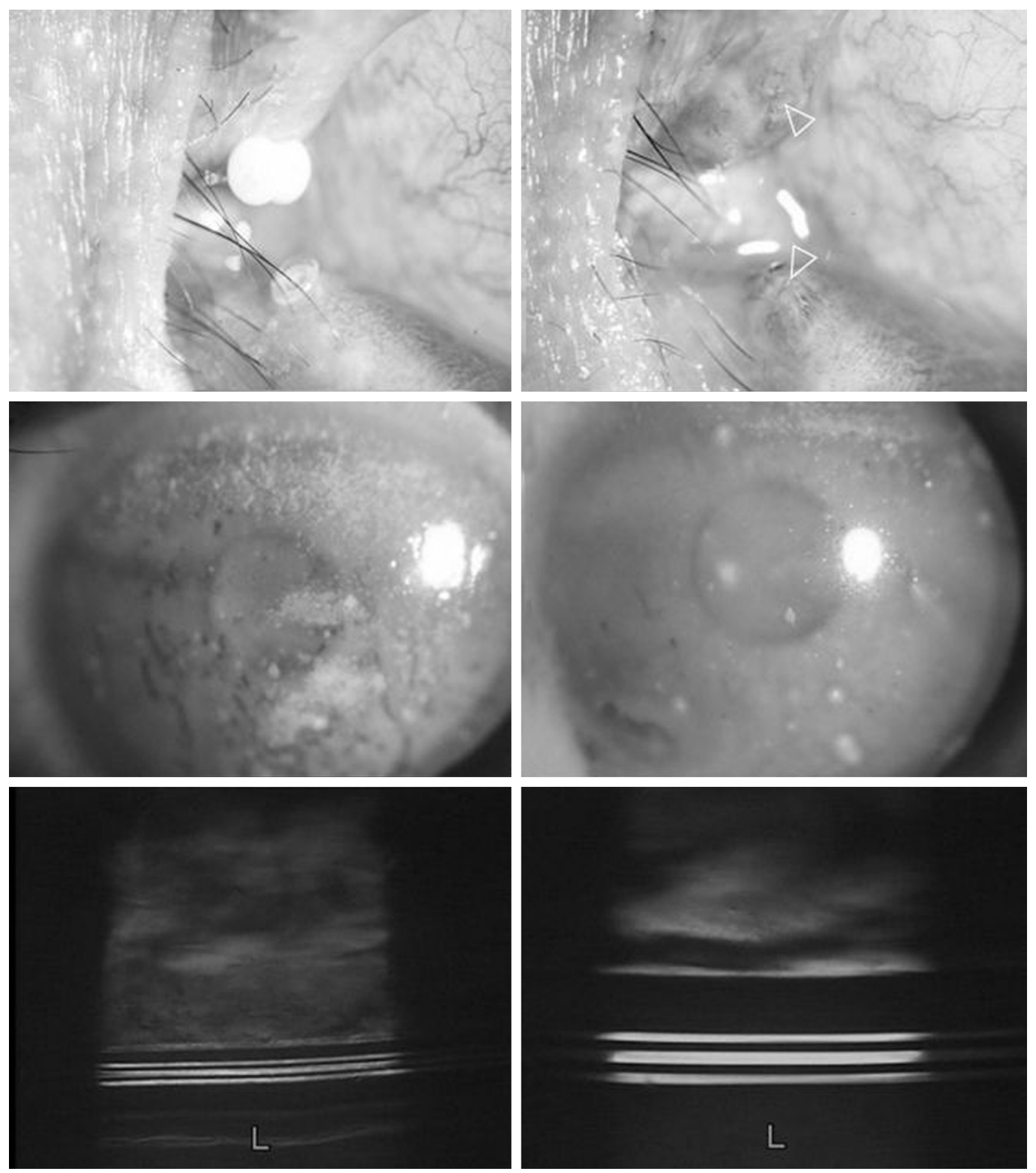

Figure 4 Representative case (Case 5 in Table I).

Notes: The left-side images show the condition of the eye prior to surgery. The top-left image shows the upper punctal plug completely extruded and hanging by sutures which were intended to fix it in the punctum. This patient experienced frequent punctal-plug extrusion in both the upper and lower puncta. The middle-left image shows the preoperative corneal epithelial damage (scored as A2D3). The bottom-left image was obtained prior to surgery by video-meniscometer (calculated tear meniscus radius [TMR]: $0.17 \mathrm{~mm}$ ). The right-side images show the condition of the eye at 6-months postoperative. The top-right image shows the puncta completely closed after undergoing our new surgical method (arrowheads). The middle-right image shows the condition of corneal epithelial damage (scored as AIDI), and the bottom-right image shows increased tear volume, as evaluated by video-meniscometer (calculated TMR: $0.36 \mathrm{~mm}$ ).

effective for long-term and complete occlusion of the punctum. At as early as 1-month postoperative, the surgically closed puncta appeared to be covered by the conjunctiva, with no inflammation around the puncta. In cases where the conjunctiva had not completely covered the sutures, they were removed when they became loose. TF analysis by meniscometry, interferometry, and measurement of FBUT, respectively, revealed that the total tear volume on the ocular surface (represented by TMR), ${ }^{22}$ the condition of the precorneal TF (represented by the IG), ${ }^{26}$ and the stability of the TF (represented by the FBUT) were improved throughout the 6-month postoperative period. We have also found that the ocular-surface staining scores, including those for fluorescein and lissamine green, were significantly improved, 

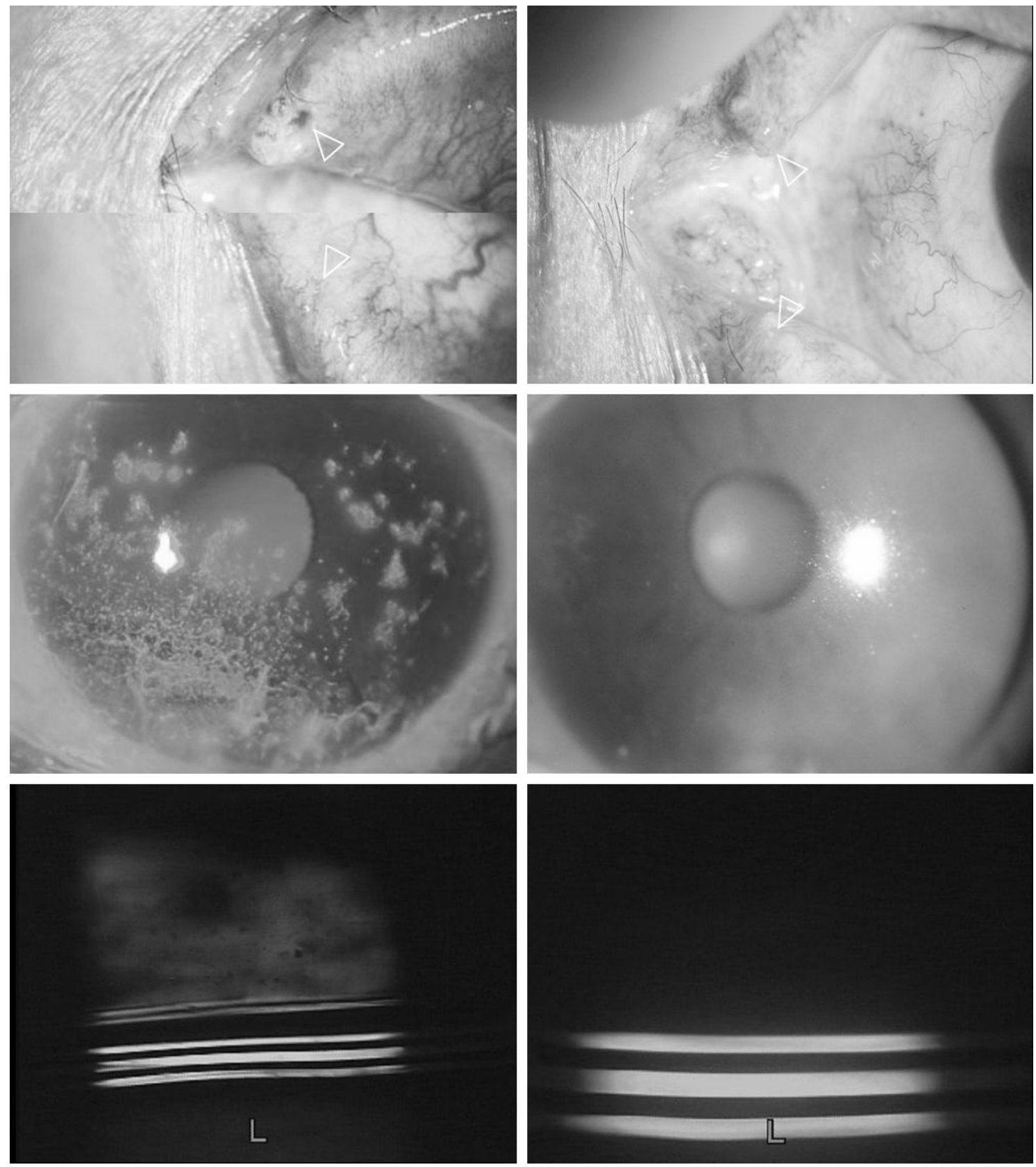

Figure 5 Representative case (Case 7 in Table I).

Notes: The upper and lower puncta of this patient had been unsuccessfully operated on four times by conventional methods of diathermy and suturing. The left-side images illustrate the preoperative condition. The top-left image shows that the upper punctum is enlarged (arrowhead) and that the lower punctum appears to have a tiny reopening (arrowhead) (Note: the upper and the lower punctum were photographed separately and adapted together for the punctum to be more clearly focused). The middle-left and bottom-left images show the condition of corneal epithelial damage (scored as A2D3) and the video-meniscometry photograph, respectively (calculated TMR: 0.33 mm). The right-side images show the condition of the eye at 6-months postoperative. The top-right image shows the puncta (arrowheads) being completely closed post our new surgical method. The middle-right image shows the condition of corneal epithelial damage (scored as A0D0), and the bottom-right image shows increased tear volume, as evaluated by video-meniscometer (calculated TMR: $0.88 \mathrm{~mm}$ ).

and that the symptoms were greatly improved in all cases throughout the 6-month postoperative period. It should be noted that continued examinations after 6-months postoperative are needed for all cases that undergo our new surgical method, but the findings in this present study appear to indicate very positive long-term improvement in tear volume, TF stability, and ocular surface epithelium without the allowance of any pin-point reopening, thus resulting in a considerable improvement of the patients' symptoms.

Comparison with the findings in another very successful study ${ }^{14}$ helped us to gauge the success of the present study. In the previous study, removal of the epithelium from the vertical portion of the canaliculus, followed by closure of the punctum with 6-0 chromic sutures, yielded a $92 \%$ success 
rate ( 24 out of 26 puncta), even after 24 months. Although the 6-month postoperative follow-up period in this present study is relatively short, the thoroughness of the examinations in our study provides very credible evidence of $100 \%$ occlusion, as well as long-term significant improvement in tear volume, TF stability, and ocular surface epithelium.

It should be noted that our new surgical procedure required the development of several new surgical tools and techniques. The first of these is a monopolar electric needle for diathermy, ${ }^{11}$ replacing the general bipolar forceps which do not always directly and efficiently cauterize the canalicular epithelium. ${ }^{2}$ In our procedure, this needle was applied gently to the canalicular wall to help the subsequent burr grinderassisted removal of epithelium. The second innovation of our new surgical procedure was the use of a hand-held motor burr grinder to remove the canalicular epithelium; the successful use of this technique has been previously reported. ${ }^{14}$ Most significantly, the third innovation, specifically aimed at the long-term stable closure of the punctum, is the use of a small amount of fibrous tissue from a part of the medial rectus $\mathrm{CPF}^{30}$ under the lacrimal caruncle. This tissue is sometimes resected during pterygium surgery with no complications. In our new surgical procedure, this tissue is inserted into the canaliculus so that it is flush with the opening of the punctum, and then fixed with anchor sutures to provide punctal occlusion without deformation.

As for other important concerns, future investigations should focus on whether or not medial rectus CPF is the optimal fibrous tissue for supporting punctal occlusion. In our preliminary trial, we used fibrous tissue from the semilunar fold, the excision of which presents no complications. ${ }^{31}$ However, this tissue is difficult to manipulate and proved to be too soft and fragile for insertion within the canaliculus. The density and consistency of fibrous tissue from the medial rectus CPF is better suited for insertion and occlusion of the punctum. We also had some concern that the use of tissue from the medial rectus CPF might lead to postoperative vision problems, since the medial rectus CPF is connected to the caruncle and helps to pull the caruncle away from the visual field during eye movement. However, none of our patients complained of any vision problems post surgery.

Of the enrolled subjects, there were numerous cases with enlarged punctum due to repeated punctal-plug extrusion. In the future, such cases can be expected to increase based on the findings in our previous report, ${ }^{4}$ which showed that the punctum size increases at an average of $0.1 \mathrm{~mm}$ each time a punctal plug is extruded. As would be expected, such cases eventually require surgical punctal occlusion when there is no appropriate-sized punctal plug available. Therefore, larger-sized puncta which have experienced repeated punctal-plug extrusion, as well as those with surgically induced deformation, may be the best candidates for our new occlusion surgery.

It should be noted that this study did have limitations, such as a relatively small sample size and short-term follow-up period. Thus, a larger sample size and careful follow-up to confirm the long-term complications such as canaliculitis and reopening of the once-closed punctum is needed, and we plan to report on those complications in a future study.

In conclusion, the postoperative findings in this study show that our newly developed surgery is both safe and effective for obtaining complete punctal occlusion, even in cases of severe ATDDE which are traditionally difficult to treat with conventional surgery. Further study is needed to simplify the surgical steps of our new punctal occlusion, ie, the use of a more appropriate burr grinder than the burr grinder for corneal rust ring to avoid the diathermy step, as well as a more appropriate and easy to manipulate suture thread than 10-0 nylon.

\section{Acknowledgments}

The authors wish to thank John Bush for reviewing the manuscript. This study was supported in part by Grants-inAid for scientific research from the Japanese Ministry of Education, Culture, Sports, Science and Technology. The authors have no proprietary or commercial interests in any of the materials discussed in this article.

\section{Disclosure}

Norihiko Yokoi is a consultant for Kissei Co. Ltd., Nagano, Japan and Rohto Co. Ltd, Osaka, Japan. Shigeru Kinoshita is a consultant for Santen Pharmaceutical Co. Ltd, Osaka, Japan and Otsuka Pharmaceutical Co. Ltd., Tokyo, Japan. Aoi Komuro and Chie Sotozono report no conflicts of interest in this work.

\section{References}

1. Diagnostic Methodology Subcommittee of the International Dry Eye Workshop (DEWS). Methodologies to diagnose and monitor dry eye disease: report of the Diagnostic Methodology Subcommittee of the International Dry Eye Workshop (2007). Ocul Surf. 2007;5(2):108-152.

2. Murube J, Murube E. Treatment of dry eye by blocking the lacrimal canaliculi. Surv Ophthalmol. 1996;40(6):463-480.

3. Baxter SA, Laibson PR. Punctal plugs in the management of dry eyes. Ocul Surf. 2004;2(4):255-266.

4. Inagaki K, Yokoi N, Nishii M, Komuro A, Kinoshita S. [Study of change of size of the punctum before insertion and after extrusion of a punctal plug and selection of an appropriate plug for reinsertion]. Nippon Ganka Gakkai Zasshi. 2005;109(5):274-278. Japanese [with English abstract]. 
5. Putterman AM. Canaliculectomy in the treatment of keratitis sicca. Ophthalmic Surg. 1991;22(8):478-480.

6. Forno E, Buzalaf F. [Removal of the vertical portion the lacrimal canaliculus in dry eye syndrome]. Arq Bras Oftalmol. 2005;68(2):199-203. Portuguese [with English abstract].

7. Kakizaki H, Takahashi Y, Iwaki M, et al. Punctal and canalicular anatomy: implications for canalicular occlusion in severe dry eye. Am J Ophthalmol. 2012;153(2):229-237.

8. Vrabec MP, Elsing SH, Aitken PA. A prospective, randomized comparison of thermal cautery and argon laser for permanent punctal occlusion. Am J Ophthalmol. 1993;116(4):469-471.

9. Knapp ME, Frueh BR, Nelson CC, Musch DC. A comparison of two methods of punctal occlusion. Am J Ophthalmol. 1989;108(3): 315-318.

10. Shalaby O, Rivas L, Rivas AI, Oroza MA, Murube J. Comparison of 2 lacrimal punctal occlusion methods. Arch Soc Esp Oftalmol. 2001; 76(9):533-536.

11. Yokoi N, Nishii M, Komuro A, Kinoshita S. [New surgical methods for punctal occlusion of severe tear-deficient dry eye and its outcome]. Nippon Ganka Gakkai Zasshi. 2004;108(9):560-565. Japanese [with English abstract].

12. Benson DR, Hemmady PB, Snyder RW. Efficacy of laser punctal occlusion. Ophthalmology. 1992;99(4):618-621.

13. DeMartelaere SL, Blaydon SM, Tovilla-Canales JL, Shore JW. A permanent and reversible procedure to block tear drainage for the treatment of dry eye. Ophthal Plast Reconstr Surg. 2006;22(5):352-355.

14. Liu D, Sadhan Y. Surgical punctal occlusion: a prospective study. Br J Ophthalmol. 2002;86(9):1031-1034.

15. Fox RI, Robinson CA, Curd JG, Kozin F, Howell FV. Sjögren's syndrome: proposed criteria for classification. Arthritis Rheum. 1986; 29(5):577-585.

16. Schirmer O. Studien zur Physiologie und Pathologie der Tränenabsonderung und Tränenabfuhr [Studies on the physiology and pathology of tear secretion and tear drainage]. Albrecht von Graefes Arch Klin. Exp Ophthalmol. 1903;56:197-291. German [with English abstract].

17. Lemp MA, Hamill JR. Factors affecting tear film breakup in normal eyes. Arch Ophthalmol. 1973;89(2):103-105.
18. Miyata K, Amano S, Sawa M, Nishida T. A novel grading method for superficial punctate keratopathy magnitude and its correlation with corneal epithelial permeability. Arch Ophthalmol. 2003;121(11):1537-1539.

19. Manning FJ, Wehrly SR, Foulks GN. Patient tolerance and ocular surface staining characteristics of lissamine green versus rose bengal. Ophthalmology. 1995;102(12):1953-1957.

20. van Bijsterveld OP. Diagnostic tests in the Sicca syndrome. Arch Ophthalmol. 1969;82(1):10-14

21. Holly FJ. Physical chemistry of the normal and disordered tear film. Trans Ophthalmol Soc UK. 1985;104(Pt 4):374-380.

22. Yokoi N, Bron AJ, Tiffany JM, Maruyama K, Komuro A, Kinoshita S. Relationship between tear volume and tear meniscus curvature. Arch Ophthalmol. 2004;122(9):1265-1269.

23. Yokoi N, Bron AJ, Tiffany JM, Kinoshita S. Reflective meniscometry: a new field of dry eye assessment. Cornea. 2000;19(3 Suppl):S37-S43.

24. Yokoi N, Bron A, Tiffany J, Brown N, Hsuan J, Fowler C. Reflective meniscometry: a non-invasive method to measure tear meniscus curvature. Br J Ophthalmol. 1999;83(1):92-97.

25. Oguz H, Yokoi N, Kinoshita S. The height and radius of the tear meniscus and methods for examining these parameters. Cornea. 2000; 19(4):497-500

26. Yokoi N, Takehisa Y, Kinoshita S. Correlation of tear lipid layer interference patterns with the diagnosis and severity of dry eye. Am J Ophthalmol. 1996;122(6):818-824.

27. Goto E, Dogru M, Kojima T, Tsubota K. Computer-synthesis of an interference color chart of human tear lipid layer, by a colorimetric approach. Invest Ophthalmol Vis Sci. 2003;44(11):4693-4697.

28. Goto E, Tseng SC. Kinetic analysis of tear interference images in aqueous tear deficiency dry eye before and after punctal occlusion. Invest Ophthalmol Vis Sci. 2003;44(5):1897-1905.

29. Law RW, Li RT, Lam DS, Lai JS. Efficacy of pressure topical anesthesia in punctal occlusion by diathermy. Br J Ophthalmol. 2005;89(11): 1449-1452.

30. Miller JM, Demer JL, Poukens V, Pavlovski DS, Nguyen HN, Rossi EA. Extraocular connective tissue architecture. J Vis. 2003;3(3):240-251.

31. Yokoi N, Komuro A, Nishii M, et al. Clinical impact of conjunctivochalasis on the ocular surface. Cornea. 2005;24(8 Suppl):S24-S31.
Clinical Ophthalmology

\section{Publish your work in this journal}

Clinical Ophthalmology is an international, peer-reviewed journal covering all subspecialties within ophthalmology. Key topics include: Optometry; Visual science; Pharmacology and drug therapy in eye diseases; Basic Sciences; Primary and Secondary eye care; Patient Safety and Quality of Care Improvements. This journal is indexed on Submit your manuscript here: http://www.dovepress.com/clinical-ophthalmology-journal

\section{Dovepress}

PubMed Central and CAS, and is the official journal of The Society of Clinical Ophthalmology (SCO). The manuscript management system is completely online and includes a very quick and fair peer-review system, which is all easy to use. Visit http://www.dovepress.com/ testimonials.php to read real quotes from published authors. 